\title{
Repetition of errors in learning and memory as a function of their prior associative strength
}

\author{
MELVIN H. MARX and KATHLEEN MARX \\ University of Missouri, Columbia, Missouri 65211
}

\begin{abstract}
In order to assess the role of prior associative strength in human learning and memory, 126 high school students were first asked to give up to four verbal responses to each of 40 word cues. Five weeks thereafter, they were given 32 words from the list as cues in a multiplechoice learning task, with response alternatives drawn from the prior responses, and 2 months later, they were given retention tests. The results in general showed reliably stronger learning and retention of errors that had been prior response associates (that is, emitted by the subject to the same cue on the 1st experimental day), compared with nonassociates. It was concluded that some place needs to be made in cognitive theories of human learning for a responsestrength concept as a complement to the learning and memory strategies customarily stressed.
\end{abstract}

Theoretical interpretations of human learning within the currently popular information processing framework show little concern for the once-dominant concept of response strength. Some of the problems raised by certain alternatives to a strictly cognitive theoretical orientation have been discussed elsewhere (Marx, 1980). The present report is an effort to provide new empirical data bearing on the role of the response-strength concept in human selective learning and memory.

A primary feature of this study is its individualized longitudinal assessment of response strength. Response associates to common words were first determined for individual subjects and were then selectively introduced as items in a typical multiple-choice learning session. Responses emitted by a subject in the first experimental sessions are called "associates" and are assumed to have a higher level of response probability, or strength, than responses not then emitted. When the associate responses occurred as first-trial errors in training their subsequent repetition in training and recurrence in retention was compared with other first-trial errors that had not been given as associates by the same subject.

This work was supported in part by a Research Career Award from the National Institute of Mental Health and a grant from the Army Research Institute for Behavioral and Social Sciences, both to the first author. The opinions expressed herein are those of the authors and do not constitute endorsement by the U.S. Army. We thank Joan Girnis and Gary Blodick, psychology teachers at Naples High School, Naples, Florida, for their cooperation in providing participants for this study and making it a part of their curriculum. Following the completion of data collection, the research design was explained and psychology as a college major and a career was discussed with the students. We also thank George Seymour for his efforts with computerized statistical analyses of the data, and Andrew Homer for his work with portions of the data.

\section{METHOD}

Subjects

A total of 126 participants, from five high school psychology classes, were involved in the study. Because they were trained and tested in their usual class setting, only those who were present during the 1 st (association-determination) day of the experiment were used for data analysis in the subsequent training and retention sessions. These analyses necessarily involved reductions in the $\mathrm{N}$, as detailed below, because of absences.

\section{Apparatus}

An Ektagraph 850 (Kodak Carousel) slide projector and various sets of $35-\mathrm{mm}$ slides with verbal cues were used for stimulus presentation. In two of the classes, we used devices providing immediate feedback by means of a stylus penetrating a template ("Rapid Raters," described in Marx \& Witter, 1972); in the other three classes, standard paper-and-pencil response techniques were employed.

\section{Design}

On Day 1, all of the 126 participants were shown a series of 40 common words and asked to provide associated words for each stimulus. From these 40 stimulus-response pairings, 32 were selected for further use; each of these cue words was paired with four responses, one of which was a highly frequent associate (from the responses earlier provided by the participants), a second of which was a moderately frequent response, a third of which was a relatively infrequent response, and the fourth of which was an idiosyncratic response (made by only one of the participants). On Day 2, this list of verbal materials was given to all of the classes, with one of the response alternatives designated as correct (randomly determined, with the restriction that each frequency category be represented equally often). On Day 3, retention tests were given, again in the usual classroom setting.

\section{Procedure}

Day 1. The 40 word cues were presented by slide projector to all of the students present in their regular classroom setting. All of the cues were standard English words. The students were instructed to respond with words that would complete a commonly used phrase. An example given was "bag" or "pole" to "bean" as a cue. Participants were instructed to write 
down, as they thought of them, up to four associated words for each cue word.

Day 2. Each class met again, within its usual setting, 5 weeks later. The list of 32 cue words, each with four response alternatives, as described above, was presented. For example, the cue "class" had response alternatives "room" (55), "rank" (1), "mate" (24), and "ring" (16). (Numbers in parentheses refer to the actual frequencies with which these words had been earlier provided by the students.) The cue "ship" had response alternatives "wreck" (32), "load" (1), "ahoy" (10), and "shape" (20). Subjects were instructed that one word alternative had been arbitrarily selected as correct for each cue and that it was their task to select as many correct words as possible. They were told that at first they would need to guess, but the correct words would be provided by one of the experimenters after the responses had been made.

Four task variations were introduced. The first two classes responded by punching one of our alternatives for each cue, utilizing the Rapid Rater device. They received immediate knowledge of results. If the stylus penetrated deeply, a correct answer was indicated; if it was stopped by the (invisible) template, an error was indicated. Six trials were planned and were administered to the second class, but apparatus malfunction (projector breakdown) permitted only three trials for the first class.

The remaining three classes were shown the stimulus materials in the same manner but used answer sheets for responses. The third class circled the appropriate number of response alternatives selected and received information as to the correct alternative as soon as all subjects had responded. The fourth class also circled the selected alternative but received all of the correct answers at the end of the trial. The fifth class wrote down their answers and received the information on correct answers at the end of each trial. The three classes were given five, seven, and six trials, respectively, the variations occurring because of time limitations in the class hours.

Day 3. Students present in the regular class period were asked to write down (1) as many of the earlier correct cueresponse word pairs as they could remember, (2) as many of the response alternatives as they could, when shown the 32 word cues by slide, and (3) as many of the correct alternatives as they could when shown both cue and four alternatives by slide. This session occurred 2 months after Day 2.

\section{RESULTS}

\section{Error Strength of Prior Associations}

The focus of this analysis is on the first-trial errors in the learning task. The experimental question addressed is: Do first-trial errors that have been identified as having relatively greater associative strength (by the individual's own prior emission of them in response to the same verbal cue) show greater persistence throughout subsequent trials than errors that have not been so identified?

Fifty participants from the three classes that used the paper-and-pencil answer procedure provided data for this analysis. They were students who were present on both the first and the second sessions. ${ }^{1}$

Overall, the total number of repetitions of first-trial prior associates was $6.3 \%(101 / 1,594)$ compared with $3.9 \%(129 / 3,304)$ for nonassociate errors. Because there is not an appropriate statistical test for these group means and there was so much variability in the scores upon which the individual ratios would be based, the chi-square test was applied to proportions of categorized individuals. With ties ignored, 30 participants were found to have a higher proportion of repetitions over Trials 2-5 for their prior associates and 16 to have a higher proportion of repetitions for their nonassociate first-trials errors. With a 50-50 theoretical expectancy baseline, this $30 / 16$ distribution of subjects yields a chi-square of 4.26 , reliable for $\mathrm{df}=1$ at $\mathrm{p}<.05$ ).

The proportion of first-trial errors that were repeated at least once was also used for the comparison of associate and nonassociate responses. This measure concentrated on whether or not each individual error was repeated at least once, rather than on the total number of error repetitions. By this measure, 19.3\% $(65 / 337)$ of the prior associate first-trial errors were repeated at least once, whereas only $11.5 \%$ (80/694) of otherwise comparable nonassociate errors were so repeated. The analysis of individual participants indicated that 26 had a higher repetition percentage for associate errors, with 8 showing the opposite result. The chi-square value of 9.53 for this distribution, again assuming a 50-50 chance expectancy, is reliable for $\mathrm{df}=1$ at $\mathrm{p}<.01$. (Subjects who did not repeat any first-trial errors were necessarily excluded from this analysis, accounting for the reduced totals.)

\section{Retention Errors}

A total of 41 students who had participated in the first two sessions in the last three classes also provided data in the final, retention, session. Because of the relatively small amount of data produced by any one of the three retention measures, a retained error was identified by its appearance on any one of the measures, and no attempt further to quantify its strength was made.

Approximately one-half $(159 / 320)$ of first-trial errors that had been prior associates were repeated in retention, whereas only $28 \%(163 / 582)$ of nonassociate first-trial errors were thus repeated. A chi-square analysis showed that the comparable difference between number of subjects showing a higher proportion of prior associate errors so retained (34) and those showing a higher proportion of nonassociate errors retained (7) was reliable, again assuming a 50-50 chance baseline $\left[\chi^{2}(1)=\right.$ $17.8, \mathrm{p}<.01]$.

An effort was made to assess the effect on error retention of error repetitions made during the learning session. The percentage of error recurrence in retention as a function of the number of error recurrence in retention as a function of the number of error occurrences in learning is shown separately for associate and nonassociate errors in Figure 1. The data base of the higher repetition values $(8 / 17$ vs. $5 / 10$ for three occurrences and $3 / 5$ vs. $5 / 8$ for four and five occurrences) is obviously too small for statistical comfort; there is nonetheless some suggestion of a progressive lessening of the difference between associate and nonassociate errors as the amount of repetition in learning increased. 


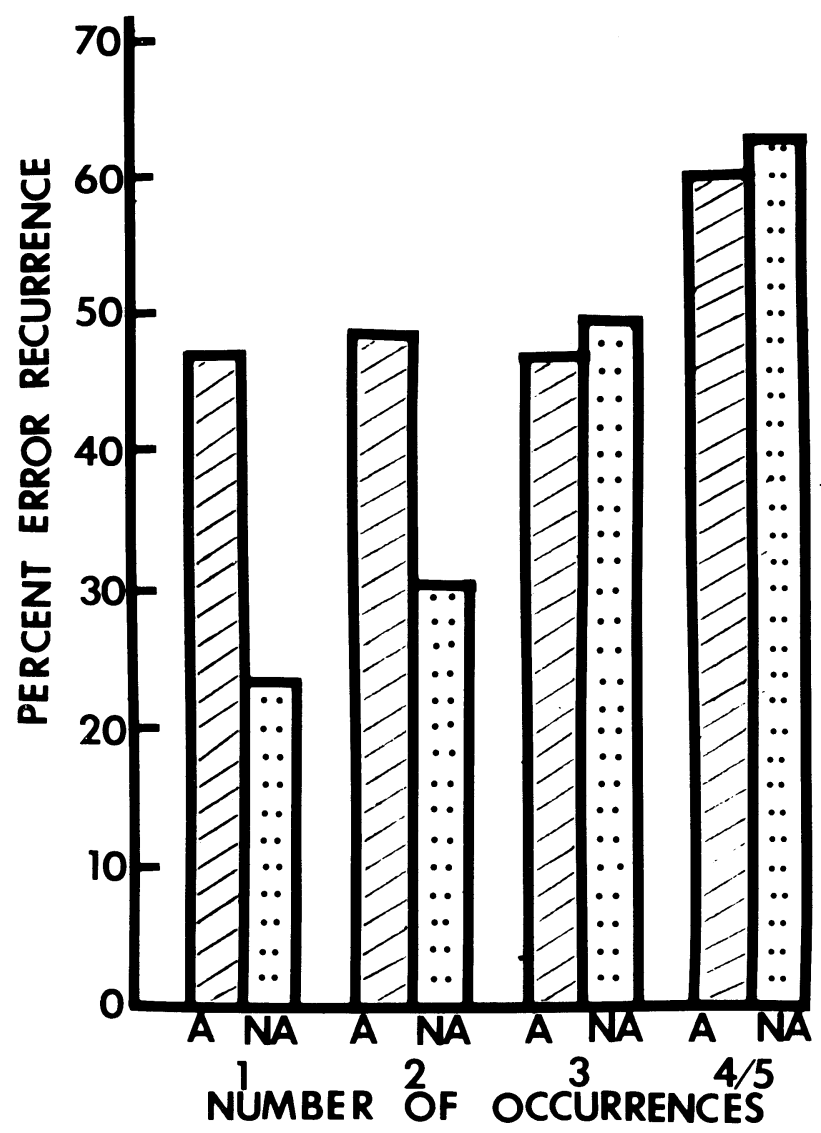

Figure 1. Percent first-trial error recurrence in retention as a function of number of occurrences in learning, for associates and nonassociates.

One final chi-square test was applied to assess the influence of practice (as indicated by error repetitions in learning) on error occurrence in retention. Retention of errors occurring only once in learning was compared with retention of errors occurring more than once. For associate errors, 11 subjects had a higher error retention score for single errors, and 18 subjects had a higher such score for multiple errors. A similar proportion was found for nonassociative errors (11 and 20, respectively). Neither of these differences in proportions reached or approached reliability ( $\chi^{2} \mathrm{~s}$ of only 1.68 and 1.58 ). (The loss of subjects in this analysis was due to omissions in one or more of the categories under comparison.)

\section{DISCUSSION}

The primary question with which this study is concerned is this: Do relatively strong prior associations have a greater probability of occurrence and persistence when subsequently made as errors in a learning task? A related, secondary question is this: Do errors persist over an extended retention period as a function of their prior associative strength and the rate of their occurrence in a learning task?

Pursuit of these questions might well seem trivial to some researchers or observers, in particular to those long steeped in stimulus-response theory and practice. It is true that the proposition at issue has often simply been assumed, but generally in the absence of specific empirical support, and especially support of a longitudinal sort. It is important to provide such support now, with so little role accorded the concept of association strength in the typical information processing stress on cognitive operations.

The present data clearly indicate some role for prior associations in error production during learning. This study also indicates the persistent influence of such prior associations over a relatively long retention period. It is difficult to see how any deliberate, conscious strategy of the type used in some of the prominent cognitive theories of human learning (e.g., Buchwald, 1969) can account for these results.

With respect to the role of the response-strength concept, a number of cognitive theorists (e.g., Fisher \& Craik, 1977) have made the reasonable point that the orthodox absolute response-strength concept cannot account for certain memory results, notably the fact that recall of the same set of events can vary markedly with type of retrieval situation (cf. Anderson \& Pichert, 1978). A response-strength concept with differentiated rather than fixed strength values, such as the habit-activation hypothesis (Marx, 1966, 1980) does not have this difficulty. By assuming that habit, in the sense of S-R potential, and habit activation, indicated by the expression of habit in performance, are separable functions, a much more flexible type of responsestrength concept can be maintained.

In our opinion, the present results point up the need for including some kind of response-strength concept in any complete account of human learning and memory. Clearly, the human subject is a superbly equipped cognitive learning agent. The real question, however, is not what the subject can do, but rather what he does do. In other words, what accounts for the failures to perform perfectly in accordance with the cognitive structures that have been established? Obviously, there are a host of conditions that contribute to errors. To name just a few, one can mention overloading of the working memory or retrieval systems, reduced or generally inadequate motivation, lapses in attention, and emotional interference of various types.

It is interesting that these conditions are more likely to obtain in real-life training and performance than they are in the typical laboratory situation, especially when adequately motivated and intellectually capable college students are the subjects. Such conditions, however, produce uncertainties in the utilization of cognitive structures and lead to errors. We need to account for such uncertainties and errors, along with the deliberate repetition of correct responses and changing of incorrect responses, in our theoretical interpretations of human learning.

\section{REFERENCES}

Anderson, R. C., \& Pichert, J. W. Recall of previously unrecallable information following a shift in perspective. Journal of Verbal Learning and Verbal Behavior, 1978, 17, 1-12.

Buchwald, A. M. Effects of "right" and "wrong" on subsequent behavior: A new interpretation. Psychological Review, 1969, 76, 132-143.

Fishe R, R. P., \& Craik, F. I. M. Interaction between encoding and retrieval operations in cued recall. Journal of Experimental Psychology: Human Learning and Memory, 1977, 3, 701-711.

Marx, M. H. The activation of habits. Psychological Reports, 1966, 19, 527-550.

MARX, M. H. Habit activation in human learning. In $\mathbf{G}$. d'Ydewalle \& W. Lens (Eds.), Cognition in human motivation and learning. New York: Academic Press, 1980.

MarX, M. H., \& WitTER, D. W. Repetition of correct responses and errors as a function of performance with reward or information. Journal of Experimental Psychology, 1972, 92, 53-58. 


\section{NOTE}

1. Data from the first two classes, utilizing the Rapid Raters, were not included in this or any subsequent analysis, because of the substantial procedural differences, the availability of only one-half (the performance part) of the total list of items, and the severely reduced number of trials in the first class. These two classes are described in this report so that they can be referred to in a subsequent report involving a different type of error analysis. Also, only the first five trials of the learning session were analyzed in order to make the scores comparable over the three classes.

(Received for publication October 30, 1980.) 